\title{
Designing cost-effective capture-recapture surveys for improving the monitoring of survival in bird populations
}

\author{
Nicolas Lieury ${ }^{1,2}$, Sébastien Devillard ${ }^{2}$, Aurélien Besnard ${ }^{3}$, Olivier Gimenez ${ }^{4}$, Olivier \\ 5 Hameau $^{5}$, Cécile Ponchon ${ }^{6} \&$ Alexandre Millon $^{1 \dagger}$ \\ 1 - Aix Marseille Univ, Univ Avignon, CNRS, IRD, IMBE, Marseille, Aix-en-Provence, \\ France \\ 2 - Laboratoire de Biométrie et Biologie Evolutive, Université de Lyon, Université Lyon 1, \\ 10 UMR5558, 69622 Villeurbanne, France \\ 3 - PSL Research University, CEFE UMR 5175, CNRS, Université de Montpellier, \\ Université Paul-Valéry Montpellier, EPHE, Biogéographie et Ecologie des Vertébrés, 1919 \\ route de Mende, 34293 Montpellier, France \\ 4 - CEFE UMR 5175, CNRS, Université Paul-Valéry Montpellier, 1919 route de Mende, \\ 1534293 Montpellier cedex 5, France. \\ 5 - Ligue pour la Protection des Oiseaux Provence-Alpes-Côtes-d'Azur, Château de \\ l'Environnement, F-84480, Buoux, France. \\ 6 - Conservatoire des Espaces Naturels Provence-Alpes-Côtes-d'Azur, Maison de la Crau, 2 \\ Place Léon Michaud, F-13310, Saint-Martin de Crau, France. \\ $\dagger-$ Corresponding author email address: alexandre.millon@imbe.fr \\ Keywords: survey design; optimisation; statistical power; cost efficiency, stage-structured \\ population \\ Running head: Cost-effective Capture-Recapture surveys
}

25 


\begin{abstract}
Population monitoring traditionally relies on population counts, accounting or not for the issue of detectability. However, this approach does not permit to go into details on demographic processes. Therefore, Capture-Recapture (CR) surveys have become popular tools for scientists and practitioners willing to measure survival response to environmental change or conservation actions. However, CR surveys are expensive and their design is often driven by the available resources, without estimation about the level of precision they provide for detecting changes in survival, despite optimising resource allocation in wildlife monitoring is increasingly important. Investigating how CR surveys could be optimised by manipulating resource allocation among different design components is therefore critically needed. We have conducted a simulation experiment exploring the statistical power of a wide range of CR survey designs to detect changes in the survival rate of birds. CR surveys differ in terms of number of breeding pairs monitored, number of offspring and adults marked, resighting effort and survey duration. We compared open-nest $(\mathrm{ON})$ and nest-box (NB) monitoring types, using medium- and long-lived model species. Increasing survey duration and number of pairs monitored increased statistical power. Long survey duration can provide accurate estimations for long-lived birds even for small population size (15 pairs). A costbenefit analysis revealed that for long-lived ON species, ringing as many chicks as possible appears as the most effective survey component, unless a technique for capturing breeding birds at low cost is available to compensate for reduced local recruitment. For medium-lived NB species, focusing the NB rounds at a period that maximises the chance to capture breeding females inside nest-boxes is more rewarding than ringing all chicks. We show that integrating economic costs is crucial when designing CR surveys and discuss ways to improve efficiency by reducing duration to a time scale compatible with management and conservation issues.
\end{abstract}


Studies aiming at detecting the response of wild populations to environmental stochasticity, anthropogenic threats or management actions (e.g. harvest, control or conservation), traditionally rely on the monitoring of population counts. Such data, however, suffers from a variable detectability of individuals that can alter the reliability of inferred temporal trends (Williams et al. 2002). Methods have been developed to account for the issue of detectability, based on the measure of the observer-animal distance (Distance Sampling; Buckland et al. 2001) or on multiple surveys (hierarchical modeling, Royle and Dorazio 2008). Still, population size being the result of a balance between survival, recruitment, emigration and immigration, inferring population status from counts, whatever detectability is accounted for or not, may impair the assignment of the demographic status of a population (source vs. sink; Furrer and Pasinelli 2016, Weegman et al. 2016).

Surveys that consist of capturing, marking with permanent tags, releasing and then recapturing wild animals (i.e. capture-recapture surveys, hereafter CR surveys), to gather longitudinal data and hence derive survival rates while accounting for imperfect detection (Lebreton et al. 1992), have become highly popular tools in both applied and evolutionary ecology (Clutton-Brock and Sheldon 2010). Opting for a mechanistic instead of a phenomenological approach has indeed proved to be particularly informative for identifying the response of a population to any perturbation, and ultimately allows to pinpoint the appropriate management strategy. Over the last decade, an increasing number of practitioners have set up CR surveys with the aim of quantifying survival variation in response to i) changing environment such as climate or habitat loss (Grosbois et al. 2008), ii) hunting (Sandercock et al. 2011), iii) other anthropogenic mortality causes (e.g. collision with infrastructures; Chevallier et al. 2015), and iv) the implementation of management/conservation actions (Lindberg 2012, Koons et al. 2013, review in Frederiksen et al. 2014). In all these contexts, the estimation of survival, and its temporal variation, is particularly informative for building effective evidence-based conservation (Sutherland et al. 2004). As an example, the high adult mortality due to electrocution in an Eagle owl Bubo bubo population of the Swiss Alps, as revealed by a CR survey, would have not been detected if the survey was solely based on population counts, that remained stable over 20 years (Schaub et al. 2010).

The effectiveness of a CR survey to detect and explain changes in survival rates over time depends on the levels of field effort dedicated to several survey components: i) the size of the sample population, ii) the proportion of offspring and adults marked, iii) the recapture/resighting rate of previously marked individuals and iv) the number of surveying years (or survey duration; Yoccoz et al. 2001, Williams et al. 2002). In a conservation context, considering only the usual trade-off between the number of marked individuals and the number of surveyed years is of little help when designing a CR survey. Indeed, practitioners need to know as soon as possible whether survival is affected by a potential threat or has alternatively benefited from a management action. Implementing CR surveys is however 90 particularly costly in terms of financial and human resources, as it requires skilled fieldworkers over an extensive time period. Therefore, most surveys are actually designed according to the level of available resources only, and without any projection about the precision they provide for estimating survival and the statistical power they obtain for detecting survival variability.

The life-history characteristics (e.g. survival and recruitment rates) of the study species largely determine which of the different components of a CR survey will provide the most valuable data. For instance, low recruitment of locally-born individuals (due to high juvenile mortality rate and/or high emigration rates) limits the proportion of individuals marked as juveniles recruited in the local population. In such a case, we expect that reducing the effort 
100 dedicated to mark offspring in favour of marking and resighting breeding individuals would improve survey efficiency. Therefore, manipulating both sampling effort and sampling design offer opportunities to optimise CR surveys. A few attempts have been made to improve the effectiveness of CR according to species' life-histories, though most of them remain speciesspecific (Devineau et al. 2006, Williams and Thomas 2009, Chambert et al. 2012, Lindberg

105 2012, Lahoz-Monfort et al. 2014). Moreover, improving CR surveys in regards to the precision of survival estimates constitutes only one side of the coin and yet, the quantification of economic costs in the optimisation process is currently lacking. Assessing costs and benefits is therefore critical if we are to provide cost-effective guidelines for designing CR surveys. This optimisation approach is increasingly considered as an important step forward

110 for improving the robustness of inferences in different contexts such as for population surveys (Moore and McCarthy 2016) or environmental DNA sampling (Smart et al. 2016).

Here we offer a simulation experiment investigating the relative efficiency of a wide array of CR survey designs in terms of statistical power to detect a change in survival rates. Alongside the usual how many and how long considerations, we focused our simulations on

115 the how to and what to monitor. We further balanced the statistical benefit of each survey component with human/financial costs, derived from actual monitoring schemes. Our aim was to provide cost-effective guidelines for the onset of new CR surveys and the improvement of existing ones. Although our work was primarily based on the monitoring of bird populations, we discussed how this approach can be applied to improve the monitoring of other taxa.

\section{Material and methods}

\subsection{Bird monitoring types and model species}

Our simulation experiment encompassed the two most common types of bird monitoring when applied on two different life-history strategies: long-lived and open-nesting species with high but delayed local recruitment $v s$. medium-lived and cavity-nesting species with rapid but low recruitment of locally-born individuals. These two types of monitoring are representative of what practitioners come across in the field and further largely determine the nature of the survey and the level of resources needed. Moreover, another prerequisite of our simulations

130 was to ensure the availability of both detailed demographic data on the model species together with a precise estimation of the human and financial costs entailed by the monitoring.

In open-nesting $(\mathrm{ON})$ surveys, chicks are typically ringed at the nest before fledging with a combination of coloured rings or a large engraved plastic ring with a simple alphanumeric code, in addition to conventional metal rings. Resightings can then be obtained without

135 recapturing the birds using binoculars or telescopes. The identification of breeding birds is typically obtained when monitoring breeding success. For our model species for ON monitoring, we combined life-history and survey characteristics of two long-lived diurnal raptors, the Bonelli's eagle Aquila fasciata and the Egyptian vulture Neophron percnopterus (Lieury et al. 2015, 2016). Monitoring typically consists of repeated visits of known territories

140 during the breeding season for checking whether breeding occurs and the identity of breeding birds, and eventually ringing chicks. Breeding birds are difficult to capture, therefore limiting the number of newly marked breeders each year, although additional trapping effort can be deployed (adults are occasionally trapped, for fitting birds with GPS). Such captures are however highly time-consuming as it requires monitoring several pre-baiting feeding stations.

The second, highly common, monitoring type concerns cavity-nesting birds, whose surveys typically involve artificial nest-boxes (NB thereafter). All NBs are checked at least once a year, and additional visits concentrate on the restricted set of occupied NBs for ringing/recapturing both chicks and breeding birds. For building simulations on the NB type of monitoring, we combined information on life-history and survey characteristics from two 
150 medium-lived nocturnal raptors, the barn owl Tyto alba (Altwegg et al. 2007) and the little owl Athene noctua ( $\mathrm{OH} \& \mathrm{AM}$, unpub. data). These two species are known to prefer NB over natural or semi-natural cavities. NB monitoring typically consists of repeated visits of NB during the breeding season for checking whether breeding occurs, catching breeding females in NB and eventually ringing chicks. Breeding females are usually relatively easy to catch, thus allowing many newly marked adults to enter the CR dataset each year, in contrast to ON. Breeding males are typically more difficult to capture than females and require alternative, time-consuming, types of trapping (Millon et al. 2010).

For the two types of monitoring, the resighting probability of non-breeding individuals (hereafter floaters) is low as such individuals are not attached to a spatially restricted nesting area. Life-cycle graphs and values of demographic parameters are given in the appendix (Table S1; Fig. S1).

\subsection{Definition of the main components of CR surveys}

165 We designed a set of surveys for both types of monitoring by varying the level of effort dedicated to four main components (Fig. S2):

1. Survey duration: For each type of monitoring, we set two different durations corresponding to 1-2 and 3-4 generations of the model species (i.e. 10/20 years and $5 / 10$ years for long- and medium-lived species respectively).

$1702 . \quad$ Number of breeding pairs surveyed: The number of pairs available for monitoring is usually lower in ON monitoring of long-lived species (with larger home-range) compared to NB monitoring of medium-lived species. Number of breeding pairs varied between 15-75 and 25-100 for ON and NB monitoring respectively.

3. Proportion of monitored nests in which chicks are ringed: This proportion was made to vary from 25 to $100 \%$ for both types of monitoring.

4. Proportion of breeders (re)captured/resighted: This proportion was set at three different levels $(0.50,0.65,0.80)$. For ON monitoring, breeding birds are not physically caught but resighted at distance. However, we evaluated the added value of a monitoring option which consists of capturing and ringing unmarked breeding adults so as to compensate for the absence of ringed adults during the early years of the survey, due to delayed recruitment in long-lived species (five adults caught every year during the first five years of the survey).

In order to reduce the number of computer-intensive simulations, we removed survey designs unlikely to be encountered in the field (e.g. only $25 \%$ of nests in which chicks are

185 ringed when 25 breeding pairs are monitored for NB). Overall, a total of 132 and 66 sampling designs were built for ON and NB monitoring respectively (Fig. S2).

\subsection{Simulating time-series of demographic rates and CR histories}

The relevance of each sampling design was assessed from 3500 simulated CR datasets. As we were interested in exploring the ability of different sampling designs to detect changes in survival, each CR dataset was generated from a survival time-series that incorporated a progressive increase in survival, mimicking the effect of conservation actions. Note here that simulating a decrease in survival would have led to similar results. The slope of the conservation effect was scaled in an additive way among ages and/or territorial status

195 according to empirical estimates from populations having benefited from conservation plans (adult survival rate increased from 0.77 to 0.88 for Bonelli's eagle, Chevalier et al. 2015; from 0.84 to 0.93 for Egyptian vulture, Lieury et al. 2015). This increase in survival rate corresponds to an increase of approximately 1.0 on the logit scale. We simulated a gradual implementation of the conservation action over the years ( 3 and 7 years for medium- and 
200 long-lived species respectively) that resulted in an increase of e.g. adult survival from 0.37 to 0.61 and from 0.81 to 0.92 for medium- and long-lived species respectively (Fig. S3). We checked the range of survival rates obtained for medium-lived species fell within the temporal variation observed in the barn owl (Altwegg et al. 2007). For each simulated CR dataset, we added random environmental variations around average survival to match variation observe in specific studies (standard deviation constant across ages on logit scale: 0.072 for ON longlived species, Lieury et al. 2015; 0.36 for NB medium-lived species, Altwegg, Schaub \& Roulin 2007). Individual CR histories were thus simulated based on survival trends (plus environmental noise) and according to the defined life-history stages (see online supplementary material for the detailed simulation procedure).

\subsection{CR analyses and contributions to statistical power}

We analysed each simulated CR dataset using a multi-state (breeder, floater) CR model for ON monitoring and a single-state model for NB monitoring (detailed structures shown in Fig. $\mathrm{S} 1$, Table S1). We then ran three models with survival i) constant $\phi_{c}$, ii) varying over years $\phi_{t}$ and iii) linearly related to the conservation action $\phi_{c o n s}$. We used ANODEV as a measure of the conservation effect on survival variation, as recommended by Grosbois et al. (2008). This statistic ensures a proper estimation of the effect of a temporal covariate whatever the level of the residual process variance. The $A N O D E V$, follows a Fisher-Snedecor distribution, and was calculated as:

$$
A N O D E V=\frac{\left(\operatorname{Dev} \phi_{c}-\operatorname{Dev} \phi_{\text {cons }}\right) /\left(k \phi_{\text {cons }}-k \phi_{c}\right)}{\left(\operatorname{Dev} \phi_{\text {cons }}-\operatorname{Dev} \phi_{t}\right) /\left(k \phi_{t}-k \phi_{c o n s}\right)}
$$

220 where $D e v$ and $k$ are, respectively, the deviance and the number of parameters of the models (Skalski et al. 1993). As a measure of the statistical power to detect a change in survival rate, we counted the number of simulations in which the ANODEV was significant. Given the limited number of years typically available in a conservation context, we chose an $\alpha$-level $=$ 0.2 to favour statistical power, at the expense of inflated probability of type I error (Yoccoz

225 1991, Grosbois et al. 2008). A specific CR survey was considered efficient when the proportion of significant ANODEV exceeded a threshold of 0.7 (Cohen 1988).

For each design, we calculated the relative increase in power by dividing the difference between the power of a given sampling design and the minimum power across all scenarios, by the difference between the maximum and minimum power across all scenarios. This ratio,

230 Apower, was used as a response variable in a linear model to quantify the effect of three explanatory variables: i) the proportion of monitored nests in which chicks are ringed, ii) the proportion of breeders (re)captured/resighted and iii) whether adult breeders were caught (in ON survey only). The survey duration and the number of surveyed nests were fixed. As explanatory variables explained $100 \%$ of the variance of $\Delta$ power, coefficients of the linear 235 model sum to 1 . Therefore, coefficients can be interpreted as the relative contribution of each design component to the increase in statistical power.

\subsection{Calculating the cost of CR surveys}

Human and financial costs of each design were derived from our own field experiences. Costs 240 included the number of working-days required to monitor a territorial pair (resighting for ON, capture/recapture for NB), to ring chicks and capture territorial breeders (for ON only). For both types of monitoring, these costs were multiplied by the number of breeding pairs surveyed, the number of monitored nests in which chicks are ringed and the total number of breeders caught. The specific case of the resighting of breeders in the ON monitoring required knowing the distribution of working-days used to check whether a given breeder was ringed and to identify it (Fig. S4). Indeed, since all territorial birds were not ringed, some observations did not provide information for the CR dataset. To account for this issue, we 
recorded from simulated demography the annual proportion of ringed breeders in the population and the number of observations. Then we calculated the costs of all bird observations, ringed or not, by sampling the number of working-days in the observed distribution of working-days (mean $=3.7 \pm 3.3$ per bird, Fig. S3). Finally, we converted the total number of working-days required for each simulation into financial cost in euros, according to the average wage of conservation practitioners in France, assuming no volunteerbased work and accounting for travel fees and supplementary materials (e.g. binocular, traps).

255 Note that we are interested in the relative, not absolute, cost of survey designs. Finally, as for statistical power, we calculated the relative contribution of the different components of a survey design to the increase of the total cost by performing a linear model with $\Delta$ costs (calculated as $\Delta$ power) as the response variable.

Finally, we calculated cost-effective contributions of each design component, by dividing the relative contribution in statistical power increase by the relative contribution in cost increase. This allowed us to specifically assess in which component one should preferentially invest to increase CR survey efficiency.

All simulations and analyses were run with $\mathrm{R} 3.1 .2$ (R Core Team 2014). We used RMark (Laake 2013) package calling program MARK (Cooch and White 2015) from $\mathrm{R}$ for CR analyses. We provided all R scripts as supplementary information (Appendices S2-S5).

\section{Results}

\subsection{Survey components affecting the power to detect a change in survival for Open- Nesting monitoring}

270 The survey duration and the number of nests surveyed were identified as the two major components for improving the ability of CR surveys in detecting a change in survival rates (Fig. 1). All long-duration surveys reached the power threshold, whereas the majority of short-duration surveys did not (44/66).

The capture of five territorial birds each year during the five first years greatly increased

275 the effectiveness of CR surveys (Fig. 1). This component actually compensated for the absence of ringed territorial birds in the early years, a consequence of delayed recruitment in long-lived species. Most survey designs lacking the initial capture of territorial birds (27/33) failed to reach the power threshold in short-duration surveys. However, the benefit in terms of statistical power of this component diminished as i) the survey duration increased from 10 to

28020 years and ii) the number of breeding pairs monitored increased. For example, when 25 breeding pairs were monitored, a survey involving the initial capture of territorial birds and $50 \%$ of nests with chicks ringed, was more efficient than a survey involving $100 \%$ of nests with chicks ringed but no territorial bird caught. Similarly, initial captures of territorial birds were more valuable than increasing the proportion of breeders resighted, although this effect

285 tended to vanish as the survey duration and/or the number of surveyed nests increased. These interactions arose from the fact that we considered an absolute number of captures, and not a fixed proportion among the birds monitored. The smaller the number of breeding pairs surveyed and the shorter the survey duration, the more valuable became the initial capture of territorial breeders. Interestingly enough, monitoring as few as 15 pairs might provide a

290 satisfactory statistical power, understanding that study has been conducted over 20 years (Fig. $1)$.

\subsection{Survey components affecting the power to detect a change in survival for Nest-Box} monitoring

295 The important environmental random variation implemented in the simulations $(\geq$ to conservation effect) produced a noisy relationship between statistical power and the level of effort dedicated to the different survey components (Fig. 2a,b). Indeed, survival of medium- 
lived species suffer from a high level of residual temporal variation, compared to long-lived species, which reduces statistical power. A solution to this issue might be found in the addition of relevant environmental covariates (e.g. prey abundance, climate indices) into CR models, to increase the ability of analyses to detect the genuine effect of conservation actions (Grosbois et al. 2008).

Trends can nevertheless be extracted and we provided an additional figure without environmental variation to ascertain these inferences (Fig. 2c,d). First, while the majority of long-duration survey reached the statistical power threshold (24/33), no sampling design did so in short-duration survey. Second, monitoring 25 pairs provided little statistical power whatever the survey duration and the level of effort dedicated to other components. Overall, the proportion of nests in which chicks were ringed had virtually no effect, partly because this component increases the proportion in the CR dataset of young birds subject to higher 310 environmental stochasticity than adults. The number of nest-boxes monitored increased statistical power and the threshold was reached for long-term survey designs including 50 nest-boxes monitored and an intermediate effort dedicated to the capture of breeding birds. The proportion of breeding birds caught appeared as the most effective component of NB surveys for medium-lived birds. This is essentially due to the fact that capturing breeding birds allowed ringing a large number of new birds, therefore enriching the CR dataset and compensating for the low recruitment rates of individuals ringed as chicks. It appeared more effective to increase the effort in terms of proportion of breeding birds caught (from 0.5 to 0.8 ), than increasing the number of pairs surveyed by 25 , especially for short-duration surveys.

\section{$320 \quad$ 3.3. Cost of CR surveys}

The number of working-days represented 97 and $88 \%$ of the total financial cost of CR surveys for ON and NB monitoring respectively. Due to the multiple visits needed to monitor breeding success, the number of nests surveyed contributed the most to the cost of CR surveys in both types of monitoring (Fig. 3). Survey duration also largely contributed to the overall costs, by multiplying this expense over the number of years (Fig. S5). In contrast, improving the recapture/resighting probability of breeders only marginally increased the survey cost. With all other things being equal, the capture of territorial birds in $\mathrm{ON}$ monitoring was more costly than improving the proportion of territorial birds resighted or increasing the proportion of chicks ringed. For NB monitoring, increasing the proportion of chicks ringed was more costly

330 than improving the recapture probability of breeders. This discrepancy between monitoring can be explained by the cost difference for a same component (Table S2): capturing a breeder in ON monitoring was much more expensive than ringing chicks (15 vs. 2 working-days), compared to NB monitoring ( 25 vs. $40 \mathrm{~min}$ ).

\subsection{The identification of cost-effective surveys}

The most efficient CR surveys were those that surveyed small numbers of nests but over long durations. However, these durations generally exceeded the timescale of management planning and did not represent an effective way to quickly adapt conservation actions in response to a threat affecting survival. Therefore, we have chosen here to focus on short340 duration surveys to identify the key design components providing the highest added value.

For ON monitoring conducted on 50 breeding pairs of a long-lived species, the most important contribution to the increase in statistical power came from the initial capture of breeding birds (29\%) but increasing the proportion of nests in which chicks are ringed proved also to be efficient ( $57 \%$ cumulated gain when passing from 25 to $100 \%$ of ringed chicks; Fig. 4a). Surprisingly, increasing the proportion of resighted territorial birds provided only limited gain of power (14\%). The contribution of these different components to the overall survey cost was highly heterogeneous with the capture of territorial breeders being particularly 
expensive (58\%), whereas ringing chicks was cheap (14\%; Fig. 4b). When balancing costs and benefits, it turned out than investing in the ringing of the chicks was the most rewarding option (Fig. 4c).

For NB monitoring conducted on 75 breeding pairs of a medium-lived species, the major contribution to the increase in statistical power was achieved through the proportion of breeding adults caught (97\% cumulative gain), with the proportion of chicks ringed providing only little added value (3\%). This trend was reinforced when considering cost contributions, such that the proportion of breeding adults caught was unambiguously pointed out as the most rewarding component of a NB sampling design (Fig. 5d,e,f).

\section{Discussion}

We offered a methodological framework for exploring the relative efficiency of alternative 360 survey designs to detect a change in survival, a key demographic parameter widely used by scientists and practitioners for monitoring animal populations. The set of sampling designs $(N$ $=198$ ) encompass the most common types of monitoring dedicated to the demographic study of birds by capture-recapture (nest-box and open-nest) and applied on medium- or long-lived species. More importantly, we conducted a cost-benefit analysis balancing the increase in

365 statistical power with costs in working-days, entailed by the four main components of CR surveys (survey duration, number of breeding pairs surveyed, proportion of monitored nests in which chicks are ringed and proportion of breeders (re)captured/resighted). For long-lived open-nesting species, increasing the proportion of chicks ringed is the most valuable option once the survey duration was fixed to a conservation-relevant timescale. In contrast, for 370 medium-lived species monitored in nest-boxes, dedicating resources to increase the proportion of breeding adults caught reduces the number of pairs monitored necessary to reach an adequate statistical power in short-duration surveys.

Our simulation experiment pointed out that extended survey durations (over 3-4 generation time) and/or high numbers of monitored breeding pairs (50-75) were often necessary to allow

375 the detection of a change in survival. This is however problematic, as long-duration surveys exceed the timescale of management planning and is unsatisfactorily regarding the implementation of conservation actions (Yoccoz et al. 2001). Moreover, practitioners dealing with species of conservation concern have to do the best of limited resources. Thus, the answers to the classical questions how long and how many are highly constrained in a

380 management context. On the one hand, practitioners need an answer as soon as possible, so as to ensure the success of the management action while limiting costs. On the other hand, the number of breeding pairs monitored is either dictated by the total number of pairs available when studying restricted populations or by the level of human/financial resources available. Overall, we believe that the questions how to and what to monitor can provide a significant added value to the design of monitoring schemes in a conservation/management context. Below we discuss several ways to overcome issues regarding monitoring design, in link with monitoring type and species life-history.

\subsection{On the relevance of ringing $100 \%$ of the offspring monitored}

390 Based on our own experience, the ability of practitioners/scientists to ring all the monitored chicks is a common quality control of CR surveys. Here we challenge this view as our simulation results showed that the validity of this 'gold standard' depends on the species' lifehistory. For long-lived species, with high recruitment of locally-born individuals, this surely constitutes a pertinent option given the low cost of this component. For species with lower local recruitment rates such as medium-lived species however, our results showed that investing in the capture of breeding adults, instead of seeking for an exhaustive ringing of chicks, is more efficient. Specifically, this strategy would consist of increasing the number of 
nest-box's rounds when breeding adults are most likely to be caught, at the expense of rounds dedicated to the ringing of the last broods.

400 It can be argued, however, that this strategy may reduce our ability to estimate juvenile survival. The population growth rate of short- and medium-lived species is theoretically more sensitive to juvenile than adult survival (e.g. Altwegg et al. 2007), although the actual contribution of different demographic traits to population dynamics may differ from theoretical expectations (e.g. Hunter et al. 2010). Therefore, it could be of prime importance to avoid CR surveys that fail in providing reliable estimates of juvenile survival for such species. Estimating juvenile survival however remains problematic (Gilroy et al. 2012). Indeed, standard CR surveys allow the estimation of apparent survival, i.e. the product between true survival and the probability of recruiting in the study area, the latter being often weak for juveniles. For NB-breeding species, apparent survival is further affected by the 410 probability of breeding in a nest-box, and not in a natural cavity where birds are typically outof-reach. Therefore, juvenile survival cannot be compared among study areas that differ by the proportion of pairs occupying nest-boxes, the latter being usually unknown. Overall, we suggest that the monitoring of new recruitment in NB survey, achieved by the capture of breeding birds, may significantly contribute to the comprehension of population dynamics in absence of reliable data on juvenile survival (Karell et al. 2009).

\subsection{Capturing breeding adults: the panacea?}

For both ON long-lived species and NB medium-lived species, the capture of breeding adults greatly improved the probability to detect a change in survival rates. Delayed recruitment in long-lived species is a major constraint to CR survey, and especially for species in which the probability to observe non-breeding birds is low. Our simulations showed that capturing some adults in the initial years greatly improved the ability of short-duration surveys to reach a satisfactory statistical power. However, the costs associated to this component vary across species and can severely reduce its effectiveness. For instance in large ON raptors, this entails prohibitive costs as it requires the mobilisation of numerous and highly-skilled people over a long time period. Alternative indirect techniques may however be implemented to reduce the costs of capturing adults (see below).

In contrast, capturing breeding birds in nest-boxes is relatively easy and cheap and only requires the knowledge of the breeding phenology. Females can be caught during late

430 incubation or when brooding chicks and therefore provide highly valuable CR data. This is especially true when considering medium-lived species in which local recruitment rate is low.

\subsection{Implementation and future directions}

If we are to reliably inform management on a reasonably short time-scale, CR surveys maximising statistical power should be favoured. Unfortunately, such surveys often include costly components such as capturing breeding individuals in ON long-lived species. Our simulations included standard CR techniques and alternative methods may be achievable to decrease the cost of the more effective but less efficient design components. For instance, collecting biological materials to implement identification of individuals through DNA

440 analyses might provide valuable data for ON long-lived species (Marucco et al. 2009, Bulut et al. 2016, Woodruff et al. 2016). Feathers of breeding birds can be searched for when nests are visited for ringing chicks. Providing that specific microsatellite markers are already available, genetic CR data can be gathered at low costs (30-50€ per sample). Alternatively, RFID microchips embedded in plastic rings may also reduce the cost of recapture by recording the ID of the parents when visiting the nests for both ON and NB (e.g. Ezard, Becker \& Coulson 2006). Reducing costs entailed by the number of nests surveyed, or the proportion of nest in 
which chicks are ringed, may be further achieved by optimising travelling costs as proposed by Moore and McCarthy (2016).

Here we took advantage of data-rich study models to set our simulations. Many species of conservation concern may lack such data but values for demographic traits can be gathered from the literature on species with similar life history characteristics. Furthermore, the effect size of the conservation effect can be set according to the extent of temporal variation in survival, as we did for the NB example. Because we did not have other systems combining a field-derived knowledge of both demographic parameters and survey costs available, we did not perform a full factorial treatment between life-history strategies and monitoring types. We believe, however, that our simulation framework enabled one to derive generic statements on the way CR surveys should be designed, partly because the relative, not absolute, costs between the different components are likely to be similar whatever the species considered. Our conclusions regarding the NB monitoring are largely insensitive to the type of life-

460 history, as the capture of breeding adults remain feasible at low cost for species with either shorter (e.g. blue tit Cyanistes caeruleus, Garcia-Navas et al. 2014) or longer life expectancy (e.g. tawny owl Strix aluco, Millon et al. 2010; Cory's shearwater Calonectris diomedea, Oppel et al. 2011). NB monitoring of passerines can entail colour ringing and resightings in addition to recapture. Regarding ON monitoring, our conclusions drawn for long-lived raptors may be altered when considering species with lower local recruitment rate and for which the capture of breeding adults, e.g. mist-nets might be easier/cheaper (e.g. ring ouzel Turdus torquatus; Sim et al. 2011). In such a case, it is likely that the cost-benefit analysis regarding the capture of adults will promote this component. Finally, many cliff-nesting seabirds show similar monitoring type and life-history characteristics to our examples and our guidelines are 470 likely to apply equally. For instance, a recent post-study evaluation of a CR survey conducted on common guillemot Uria aalge found that resighting effort could be halved without altering the capacity to monitor survival (Lahoz-Monfort et al. 2014), in agreement with our results. The complete R scripts provided as electronic supplements can be modified to help designing specific guidelines for other species.

475 Finally, the different components of CR design considered in our simulations are somewhat specific to bird ecology and may not directly apply when considering other vertebrates such as mammals, reptiles or amphibians. For instance, in carnivorous mammals, CR surveys are limited by the difficulty of capturing/recapturing individuals with elusive behaviour. Survival estimations often rely on the use of GPS/VHF tracking that is not well suited for long-term 480 monitoring. Camera-trapping and DNA-based identification are increasingly used to improve CR surveys in such species (Marucco et al. 2009, Cubaynes et al. 2010, O'Connell et al. 2010) and we believe that a cost-efficiency approach may be helpful for carefully designing optimal surveys in such monitoring. For example, one could simulate different sampling designs varying by trap number, inter-trap distance and the area covered for carnivores having small or large home-ranges to assess the effect of these components on the detection of survival variation. The path is, therefore, open for developing cost-effective CR surveys and improving the output of wildlife monitoring in all management situations.

\section{Acknowledgments}

490 We would like to thank all the practitioners we have worked with for sharing their experiences on the monitoring of wild populations. NL received a PhD Grant from École Normale Supérieure/EDSE Aix-Marseille Université. Comments from two anonymous reviewers helped us to improve the quality of the manuscript. Sonia Suvelor kindly edited the English. 


\section{References}

Altwegg, R., M. Schaub, and A. Roulin. 2007. Age-specific fitness components and their temporal variation in the barn owl. The American naturalist 169:47-61.

Buckland, S. T., D. R. Anderson, K. P. Burnham, J. L. Laake, D. L. Borchers, and L. Thomas. 2001. Introduction to Distance Sampling. Estimating abundance of biological populations. Oxford University Press, Oxford, UK.

Bulut, Z., E. Bragin, A. DeWoody, M. Braham, T. Katzner, and J. Doyle. 2016. Use of Noninvasive Genetics to Assess Nest and Space Use by White-tailed Eagles. Journal of Raptor Research 50:351-362.

Chambert, T., D. Pardo, R. Choquet, V. Staszewski, K. D. McCoy, T. Tveraa, and T. Boulinier. 2012. Heterogeneity in detection probability along the breeding season in Black-legged Kittiwakes: Implications for sampling design. Journal of Ornithology 152:371-380.

Chevallier, C., A. Hernandez-Matias, R. Joan, N. Vincent-Martin, A. Ravayrol, and A. Besnard. 2015. Retrofitting of power lines effectively reduces mortality by electrocution in large birds: an example with the endangered Bonelli's eagle. Journal of Applied Ecology 52:1465-1473.

Clutton-Brock, T., and B. C. Sheldon. 2010. Individuals and populations: the role of longterm, individual-based studies of animals in ecology and evolutionary biology. Trends in Ecology \& Evolution 25:562-73.

Cohen, J. 1988. Statistical Power Analysis for the Behavioral Sciences. Second. Lawrence Erlbaum Associates, Hillsdale, New Jersey, U.S.

Cooch, E., and G. White. 2015. Program MARK: a gentle introduction. Free download at http://www. phidot. org/software/mark. 14th edition.

Cubaynes, S., R. Pradel, R. Choquet, C. Duchamp, J.-M. Gaillard, J.-D. Lebreton, E. Marboutin, C. Miquel, A.-M. Reboulet, C. Poillot, P. Taberlet, and O. Gimenez. 2010. Importance of accounting for detection heterogeneity when estimating abundance: the case of French wolves. Conservation Biology 24:621-626.

Devineau, O., R. Choquet, and J.-D. Lebreton. 2006. Planning Capture-Recapture Studies: Straightforward Precision, Bias, and Power Calculations. Wildlife Society Bulletin 34:1028-1035.

Ezard, T. H. G., P. H. Becker, and T. Coulson. 2006. The contributions of age and sex to variation in common tern population growth rate. Journal of Animal Ecology 75:13791386.

Frederiksen, M., J.-D. Lebreton, R. Pradel, R. Choquet, and O. Gimenez. 2014. Identifying links between vital rates and environment: a toolbox for the applied ecologist. Journal of Applied Ecology 51:71-81.

Furrer, R. D., and G. Pasinelli. 2016. Empirical evidence for source-sink populations: a review on occurrence, assessments and implications. Biological Reviews 91:782-795.

Garcia-Navas, V., E. S. Ferrer, J. J. Sanz, and J. Ortego. 2014. The role of immigration and local adaptation on fine-scale genotypic and phenotypic population divergence in a less mobile passerine. Journal of Evolutionary Biology 27:1590-1603.

Grosbois, V., O. Gimenez, J. M. Gaillard, R. Pradel, C. Barbraud, J. Clobert, A. P. Moller, and H. Weimerskirch. 2008. Assessing the impact of climate variation on survival in vertebrate populations. Biological Reviews 83:357-399. 
Karell, P., K. Ahola, T. Karstinen, A. Zolei, and J. E. Brommer. 2009. Population dynamics in a cyclic environment: consequences of cyclic food abundance on tawny owl reproduction and survival. Journal of Animal Ecology 78:1050-1062.

Koons, D. N., R. F. Rockwell, and L. M. Aubry. 2013. Effects of exploitation on an overabundant species: the lesser snow goose predicament. Journal of Animal Ecology 83:365-374.

Laake, J. L. 2013. RMark: An R Interface for Analysis of Capture-Recapture Data with MARK.

Lahoz-Monfort, J. J., M. P. Harris, B. J. T. Morgan, S. N. Freeman, and S. Wanless. 2014. Exploring the consequences of reducing survey effort for detecting individual and temporal variability in survival. Journal of Applied Ecology 51:534-543.

Lebreton, J.-D., K. P. Burnham, J. Clobert, and D. R. Anderson. 1992. Modeling survival and testing biological hypotheses using marked animals: a unified approach with case studies. Ecological Monographs 62:67-118.

555 Lieury, N., A. Besnard, C. Ponchon, A. Ravayrol, and A. Millon. 2016. Geographically isolated but demographically connected: Immigration supports efficient conservation actions in the recovery of a range-margin population of the Bonelli's eagle in France. Biological Conservation 195:272-278.

Lieury, N., M. Gallardo, C. Ponchon, A. Besnard, and A. Millon. 2015. Relative contribution of local demography and immigration in the recovery of a geographically-isolated population of the endangered Egyptian vulture. Biological Conservation 191:349-356.

Lindberg, M. S. 2012. A review of designs for capture - mark - recapture studies in discrete time. Journal of Ornithology 152:S355-S370.

Marucco, F., D. H. Pletscher, L. Boitani, M. K. Schwartz, K. L. Pilgrim, and J.-D. Lebreton. 2009. Wolf survival and population trend using non-invasive capture-recapture techniques in the Western Alps. Journal of Applied Ecology 46:1003-1010.

Millon, A., S. J. Petty, and X. Lambin. 2010. Pulsed resources affect the timing of first breeding and lifetime reproductive success of tawny owls. Journal of Animal Ecology 79:426-435.

Moore, A. L., and M. A. McCarthy. 2016. Optimizing ecological survey effort over space and time. Methods in Ecology and Evolution 7:891-899.

O’Connell, A. F., J. D. Nichols, and K. U. Karanth. 2010. Camera traps in animal ecology: methods and analyses. Springer, Tokyo.

Oppel, S., A. F. Raine, J. J. Borg, H. Raine, E. Bonnaud, K. Bourgeois, A. Breton, and D. R. 2011. Is the Yelkouan shearwater Puffinus yelkouan threatened by low adult survival probabilities? Biological Conservation 144:2255-2263.

R Core Team. 2014. R: A language and environment for statistical computing. R Foundation for Statistical Computing, Vienna, Austria.

Royle, J. A. R., and R. M. Dorazio. 2008. Hierarchical Modeling and Inference in Ecology: the Analysis of Data from Populations, Metapopulations and Communities. Academic Press.

Sandercock, B. K., E. B. Nilsen, H. Brøseth, and H. C. Pedersen. 2011. Is hunting mortality additive or compensatory to natural mortality? Effects of experimental harvest on the survival and cause-specific mortality of willow ptarmigan. Journal of Animal Ecology 80:244-258. 
Schaub, M., A. Aebischer, O. Gimenez, S. Berger, and R. Arlettaz. 2010. Massive immigration balances high anthropogenic mortality in a stable eagle owl population: Lessons for conservation. Biological Conservation 143:1911-1918.

Sim, I. M. W., G. W. Rebecca, S. C. Ludwig, M. C. Grant, and J. M. Reid. 2011. Characterizing demographic variation and contributions to population growth rate in a declining population. Journal of Animal Ecology 80:159-170.

Skalski, J. R., A. Hoffmann, and S. G. Smith. 1993. Testing the significance of individual- and cohort-level covariates in animal survival studies. Pages 9-28 in J.-D. Lebreton and P. M. North, editors. Marked Individuals in the Study of Bird Population. BirkhäuserVerlag, Basel, Switzerland.

Smart, A. S., A. R. Weeks, A. R. van Rooyen, A. Moore, M. A. McCarthy, and R. Tingley. 2016. Assessing the cost-efficiency of environmental DNA sampling. Methods in Ecology and Evolution 7:1291-1298.

Sutherland, W. J., A. S. Pullin, P. M. Dolman, and T. M. Knight. 2004. The need for evidencebased conservation. Trends in Ecology \& Evolution 19:305-308.

Weegman, M. D., S. Bearhop, A. D. Fox, G. M. Hilton, A. J. Walsh, J. L. McDonald, and D. J. Hodgson. 2016. Integrated population modelling reveals a perceived source to be a cryptic sink. Journal of Animal Ecology 85:467-475.

Williams, B. K., J. D. Nichols, and M. J. Conroy. 2002. Analysis and Management of Animal Populations: Modeling, Estimation, and Decision Making. Academic Press, San Diego, USA.

Williams, R., and L. Thomas. 2009. Cost-effective abundance estimation of rare animals: Testing performance of small-boat surveys for killer whales in British Columbia. Biological Conservation 142:1542-1547.

610 Woodruff, S. P., P. M. Lukacs, D. Christianson, and L. P. Waits. 2016. Estimating Sonoran pronghorn abundance and survival with fecal DNA and capture - recapture methods. Conservation Biology 30:1102-1111.

Yoccoz, N. G. 1991. Use, overuse, and misuse of significance tests in evolutionary biology and ecology. Bulletin of the Ecological Society of America 72:106-111.

615 Yoccoz, N. G., J. D. Nichols, and T. Boulinier. 2001. Monitoring of biological diversity in space and time. Trends in Ecology and Evolution 16:446-453. 